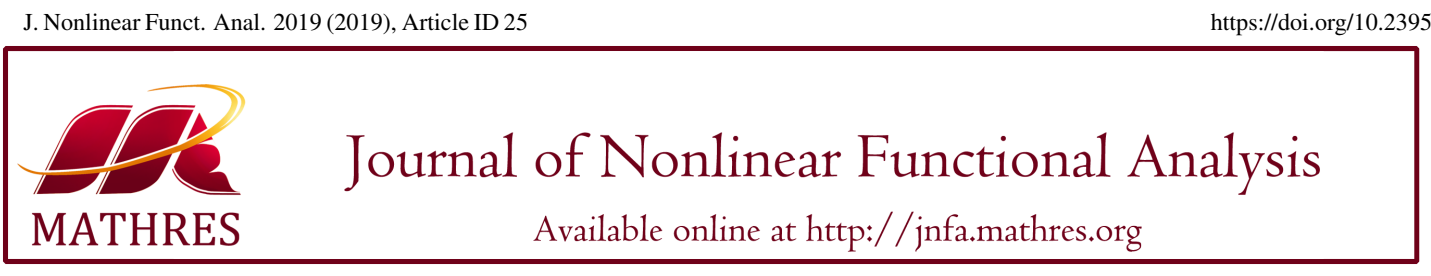

\title{
A PRIMAL-DUAL INTERIOR-POINT METHOD FOR THE SEMIDEFINITE PROGRAMMING PROBLEM BASED ON A NEW KERNEL FUNCTION
}

\author{
IMENE TOUIL $^{1, *}$, DJAMEL BENTERKI ${ }^{2}$ \\ ${ }^{1}$ LMAM, Department of Mathematics, Mohammed Seddik Ben Yahia University, Algeria \\ ${ }^{2}$ LMFN, Department of Mathematics, Ferhat Abbas University, Algeria
}

\begin{abstract}
The purpose of this paper is to improve the complexity of a large-update primal-dual interior point method for a semidefinite programming problem. We define a proximity function for the semidefinite programming problem based on a new parametric kernel function and prove that the worst-case iteration bound for the new correspondent algorithm is $\mathscr{O}\left(\sqrt{n}(\ln n)^{\frac{p q+1}{p q}} \ln \frac{n}{\varepsilon}\right)$, where $p, q \geq 1$.
\end{abstract}

Keywords. Linear semidefinite programming; Primal-dual interior point methods; Kernel function; Complexity analysis; Worst-case iteration bound.

2010 Mathematics Subject Classification. 90C22, 90C51.

\section{INTRODUCTION}

Interior point methods (IPMs) were initiated by Narendra Karmarkar in 1984 [1] are now among the most effective methods for solving linear programming (LP) problems. They not only have polynomial complexity, but also are highly efficient in practice. They motivate researchers to elaborate extensions for more general classes of optimization problems: the linear complementarity problem (LCP) [2], the convex programming problem $(\mathrm{CP})[3]$ and the semidefinite programming problem (SDP) $[4,5,6]$.

Most IPMs for LP and SDP are based on the logarithmic barrier function [7, 8]. Recently, Peng, Roos and Terlaky $[9,10]$ designed primal-dual IPMs by using self regular functions for LP and extended the approach to SDP. The complexity bounds obtained by them are $\mathscr{O}\left(\sqrt{n} \ln n \ln \frac{n}{\varepsilon}\right)$ and $\mathscr{O}\left(\sqrt{n} \ln \frac{n}{\varepsilon}\right)$ for largeupdate methods and small-update methods, respectively, where $n$ is the size of the problem and $\varepsilon$ is the accuracy parameter. They are now the best known iteration bounds. At present, the best known theoretical iteration bound for small-update method is better than the one for large-update method. However, large-update methods are much more efficient than small-update methods in practice; see [8, 11, 12]. In 2005, Wang, Bai and Roos [6] presented a primal-dual interior point algorithm for SDP problems based

${ }^{*}$ Corresponding author.

E-mail addresses: i_touil@yahoo.fr (I. Touil), djbenterki@univ-setif.dz (D. Benterki).

Received January 25, 2019; Accepted June 17, 2019.

(C)2019 Journal of Nonlinear Functional Analysis 
on a simple non self-regular kernel function, which was first introduced in [13] for LP. They derived that the complexity bounds for large and small-update methods are $\mathscr{O}\left(q n \ln \frac{n}{\varepsilon}\right)$ and $\mathscr{O}\left(q^{2} \sqrt{n} \ln \frac{n}{\varepsilon}\right)$, respectively, which are as good as those in linear case. Later on, Qian, Bai and Wang [14] proposed a new kernel function with simple algebraic expression and established the iteration complexity as $\mathscr{O}\left(n^{\frac{3}{4}} \ln \frac{n}{\varepsilon}\right)$ for large-update method for SDP. In 2009, Choi and Lee [12] proposed a new kernel function based on a new proximity function for SDP and established that the worst-case iteration bound is $\mathscr{O}\left(\sqrt{n}(\ln n)^{\frac{q+1}{q}} \ln \frac{n}{\varepsilon}\right)$ for large-update IPMs. Since then, many various kernel functions have been proposed and analyzed. For these, we refer the readers to $[11,15,16,17,18,19,20,21]$ for both linear and semidefinite cases. The key of all these results is to find kernel functions that improve the complexity analysis of LP and SDP problems.

In this paper, motivated by the above results, we introduce a new parametric kernel function with the exponential barrier term and propose a new IPM for SDP based on this kernel function. We develop some new analytic tools that are used in the complexity analysis of the algorithm. Finally, we obtain that the worst-case iteration bound for large-update methods is $\mathscr{O}\left(\sqrt{n}(\ln n)^{\frac{p q+1}{p q}} \ln \frac{n}{\varepsilon}\right)$. This parametric kernel function yields the similar complexity bound given in $[7,12,18,21]$. For small-update methods, we obtain the best known iteration bound, namely, $\mathscr{O}\left(\sqrt{n} \ln \frac{n}{\varepsilon}\right)$.

The paper is organized as follows. In Section 2, we describe special matrix functions and briefly review the basic concepts of IPMs for SDP. In Section 3, we define a new parametric kernel function $\psi(t)$ and give its properties. The generic primal-dual interior point algorithm based on our kernel function for the SDP is presented in Section 4. The complexity analysis of the algorithm is performed in Section 5. In Section 6, we offer a compared results of the algorithm given by Choi and Lee [12] and our algorithm. Finally, some conclusions and remarks are given in the last section.

Some notations used throughout the paper are as follows. The set of all $(n \times n)$ matrices with real entries is denoted by $\mathbb{R}^{n \times n}$. Given $M \in \mathbb{R}^{n \times n}, M^{t}$ denotes the transpose of $M$. $\mathbb{S}_{+}^{n}\left(\mathbb{S}_{++}^{n}\right)$ denotes the cone of positive semidefinite (positive definite) matrices in the real space of $(n \times n)$ symmetrical matrices $\mathbb{S}^{n}$. The scalar product of two matrices $A$ and $B$ in $\mathbb{S}^{n}$ is the trace of their product, i.e., $\langle A, B\rangle=\operatorname{tr}(A B)=$ $\sum_{i, j=1}^{n} a_{i j} b_{i j}$. For any $M \in \mathbb{S}^{n}$, we denote by $\lambda_{i}(M), i=1, \ldots, n, \lambda_{\max }(M)$ and $\lambda_{\min }(M)$ the eigenvalues, the largest eigenvalue and the smallest eigenvalue of the matrix $M$. The diagonal matrix with diagonal entries $\lambda_{i}(M), i=1, \ldots n$, is denoted by $\operatorname{diag}\left(\lambda_{1}(M), \ldots, \lambda_{n}(M)\right)$. The Frobenius norm of $M \in \mathbb{S}^{n}$ is $\|M\|=$ $\langle M, M\rangle^{\frac{1}{2}}=\sqrt{\sum_{i=1}^{n} \lambda_{i}^{2}(M)}$. For $M \in \mathbb{S}^{n}, M \succeq 0(M \succ 0)$ means that $M$ is a positive semidefinite (positive definite) matrix. For any $M \in \mathbb{S}_{++}^{n}$, the expression $M^{\frac{1}{2}}$ (or $\sqrt{M}$ ) denotes its symmetric square root. Finally, if $f(x) \geq 0$ is a real valued function of a real nonnegative variable, the notation $f(x)=\mathscr{O}(x)$ means that $f(x) \leq C x$ for some positive constant $C$ and $f(x)=\Theta(x)$ means that $C_{1} x \leq f(x) \leq C_{2} x$ for two positive constants $C_{1}$ and $C_{2}$.

\section{PRELIMINARIES}

2.1. Matrices and matrix functions. To introduce matrix functions which will be useful for designing the primal-dual interior point algorithm, let first us recall some known facts from linear algebra. For more details, we refer to the books [22, 23, 24]. 
Theorem 2.1. (Spectral theorem) Let $A \in \mathbb{R}^{n \times n}$ be a matrix. A is a symmetric if and only if there exists an orthogonal matrix $U \in \mathbb{R}^{n \times n}\left(U^{t} U=I\right)$ such that $U^{t} A U=\operatorname{diag}\left(\lambda_{1}(A), \ldots, \lambda_{n}(A)\right)$, where $I$ is the $(n \times n)$ unit matrix.

The columns $u_{i}$ of $U$ are the eigenvectors of $A$ satisfying

$$
A u_{i}=\lambda_{i}(A) u_{i}, \quad i=1, \ldots, n .
$$

Now, we are ready to show how a matrix function can be obtained from $\psi(t)$, where $\psi(t)$ is a real function on $[0,+\infty[$.

Definition 2.2. Let $V \in \mathbb{S}_{++}^{n}$ and

$$
V=Q^{t} \operatorname{diag}\left(\lambda_{1}(V), \lambda_{2}(V), \ldots, \lambda_{n}(V)\right) Q,
$$

where $Q$ is any orthonormal matrix $\left(Q^{t}=Q^{-1}\right)$ that diagonalizes $V$. Let $\psi(t)$ be a real function on $\left[0,+\infty\left[\right.\right.$. The matrix function $\psi(V): \mathbb{S}_{++}^{n} \rightarrow \mathbb{S}^{n}$ is defined by

$$
\psi(V)=Q^{t} \operatorname{diag}\left(\psi\left(\lambda_{1}(V)\right), \psi\left(\lambda_{2}(V)\right), \ldots, \psi\left(\lambda_{n}(V)\right)\right) Q .
$$

It should be noted that matrix $Q$ is not unique, but $\psi(V)$ is well defined whenever $\psi(t)$ is well defined on the eigenvalues of $V$.

Remark 2.3. If the function $\psi(t)$ is differentiable on the interval $] 0,+\infty\left[\right.$ such that $\psi^{\prime}(t)>0, \forall t>0$, we can obtain the matrix function $\psi^{\prime}(V)$ if $\psi\left(\lambda_{i}(V)\right)$ in $(2.1)$ is replaced by $\psi^{\prime}\left(\lambda_{i}(V)\right)$ for each $i$.

Definition 2.4. [12] The proximity function (measure) for the SDP is defined as follows

$$
\Phi(X, S ; \mu):=\Psi(V):=\operatorname{tr}(\psi(V))=\sum_{i=1}^{n} \psi\left(\lambda_{i}(V)\right),
$$

where $\psi(V)$ is defined by $(2.1)$.

Proposition 2.5. [12] For any $X_{1} \succ 0$ and $X_{2} \succ 0$, we have

$$
\Psi\left(\left(X_{1}^{\frac{1}{2}} X_{2} X_{1}^{\frac{1}{2}}\right)^{\frac{1}{2}}\right) \leq \frac{1}{2}\left(\Psi\left(X_{1}\right)+\Psi\left(X_{2}\right)\right) .
$$

Definition 2.6. A matrix $M(t)$ is said to be a matrix of function if each entry of $M(t)$ is a function of $t$, i.e., $M(t)=\left[M_{i j}(t)\right]$.

The usual concepts of continuity, differentiability and integrability can be naturally extended to matrices of functions, by interpreting them entry-wise. Then, it can easily be understood that

$$
\frac{d}{d t}(M(t)):=\left[\frac{d}{d t} M_{i j}(t)\right]=M^{\prime}(t) .
$$

Suppose that the matrix-valued functions $M(t), N(t)$ are differentiable with respect to $t$. Then

and

$$
\begin{aligned}
& \frac{d}{d t}(\operatorname{tr}(M(t)))=\operatorname{tr}\left(\frac{d}{d t} M(t)\right)=\operatorname{tr}\left(M^{\prime}(t)\right), \\
& \frac{d}{d t} \operatorname{tr}(\psi(M(t)))=\operatorname{tr}\left[\psi^{\prime}(M(t))\left(M^{\prime}(t)\right)\right]
\end{aligned}
$$

$$
\frac{d}{d t}(M(t) N(t))=\left(\frac{d}{d t} M(t)\right) N(t)+M(t)\left(\frac{d}{d t} N(t)\right)=M^{\prime}(t) N(t)+M(t) N^{\prime}(t) .
$$


2.2. The central path. We consider the SDP problem in the standard form

$$
(P)\left\{\begin{array}{c}
\min \langle C, X\rangle \\
\mathscr{A} X=b, \\
X \in \mathbb{S}_{+}^{n},
\end{array}\right.
$$

and its dual problem

$$
\text { (D) }\left\{\begin{array}{l}
\max b^{t} y \\
\mathscr{A}^{*} y+S=C, \\
S \in \mathbb{S}_{+}^{n},
\end{array}\right.
$$

where each $A_{i}$ in $\mathbb{S}^{n}, b, y \in \mathbb{R}^{m}, C \in \mathbb{S}^{n}, \mathscr{A}$ is a linear operator from $\mathbb{S}^{n}$ to $\mathbb{R}^{m}$ defined by

$$
\mathscr{A} X=\left(\left\langle A_{1}, X\right\rangle,\left\langle A_{2}, X\right\rangle, \ldots,\left\langle A_{m}, X\right\rangle\right)^{t}
$$

and $\mathscr{A}^{*}$ is the adjoint of $\mathscr{A}$ defined from $\mathbb{R}^{m}$ to $\mathbb{S}^{n}$ by $\mathscr{A}^{*} y=\sum_{i=1}^{m} y_{i} A_{i}$.

Throughout this paper, we assume that both $(P)$ and $(D)$ satisfy the interior point condition $(I P C)$, that is, there exists $\left(X^{0} \succ 0, y^{0}, S^{0} \succ 0\right)$ such that

$$
\mathscr{A} X^{0}=b, X^{0} \succ 0, \mathscr{A}^{*} y^{0}+S^{0}=C, S^{0} \succ 0 .
$$

We also assume that the matrices $A_{i}, i=1, \ldots, m$, are linearly independent. It is well known that the IPC can be assumed without loss of generality. In fact, we may choose $X^{0}=S^{0}=I$ as the initial start point. For the details, we refer to the book [24].

The Karush-Kuhn-Tucker (KKT) conditions for $(P)$ and $(D)$ are given by

$$
\left\{\begin{array}{l}
\mathscr{A} X=b, X \succ 0, \\
\mathscr{A}^{*} y+S=C, S \succ 0, \\
X S=0 .
\end{array}\right.
$$

The core idea of primal-dual IPMs is to replace the third equation in (2.7), the so-called complementarity condition for $(P)$ and $(D)$ by the parameterized equation $X S=\mu I$, with $\mu>0$. This yields the following system

$$
\left\{\begin{array}{l}
\mathscr{A} X=b, X \succ 0, \\
\mathscr{A}^{*} y+S=C, S \succ 0, \\
X S=\mu I, \quad \mu>0 .
\end{array}\right.
$$

Under the IPC, system (2.8) has a unique solution denoted by $\left(X_{\mu}, y_{\mu}, S_{\mu}\right)$, for each $\mu>0$. The set of all solutions $\left(X_{\mu}, y_{\mu}, S_{\mu}\right)$ with $\mu>0$ is known as the central path or central trajectory of $(P)$ and $(D)$. If $\mu \rightarrow 0$, then the limit of the central path exist. Since the limit points satisfy the complementarity condition, the limit yields optimal solutions for $(P)$ and $(D)$ (see, e.g., $[5,24]$ ).

2.3. The search direction. The Newton's method is a well known procedure to solve a system of nonlinear equations. Most IPMs for solving the SDP employ different search directions together with suitable strategies for following the central path appropriately. Without loss of generality, we assume that $(X(\mu), y(\mu), S(\mu))$ is known for some positive $\mu$. Then we decrease $\mu$ to $\mu=(1-\theta) \mu$ for some fixed 
$\theta \in(0,1)$. Applying Newton's method to system (2.8) for getting approximate solutions, we have the following linear system

$$
\left\{\begin{array}{l}
\mathscr{A} \Delta X=b \\
\mathscr{A}^{*} \Delta y+\Delta S=C \\
\Delta X S+X \Delta S=\mu I-X S
\end{array}\right.
$$

where $(\Delta X, \Delta y, \Delta S)$ is the search direction. It is obvious that $\Delta S$ is symmetric due to the second equation in (2.9). However, a crucial observation is that $\Delta X$ is not necessarily symmetric because the third equation of system (2.9) may not be symmetric; see [6]. There are several methods for symmetrizing the third equation in the Newton system such that the new system has a unique symmetric solution; see $[25,26,27]$.

In this paper, we consider the Nesterov-Todd (NT)-symmetrization scheme in [26]. One important reason for this is that the NT scaling technique transfers the primal variable $X$ and the dual variable $S$ into the same space: the so-called $V$-space. Let us define the matrix

$$
P=\left[X^{\frac{1}{2}}\left(X^{\frac{1}{2}} S X^{\frac{1}{2}}\right)^{-\frac{1}{2}} X^{\frac{1}{2}}\right]^{-\frac{1}{2}}=\left[S^{-\frac{1}{2}}\left(S^{\frac{1}{2}} X S^{\frac{1}{2}}\right)^{\frac{1}{2}} S^{-\frac{1}{2}}\right]^{-\frac{1}{2}} .
$$

Furthermore, we define $D=P^{-1}$. The matrix $D$ can be used to scale $X$ and $S$ to the same matrix $V$ because

$$
V=\frac{1}{\sqrt{\mu}} D^{-1} X D^{-1}=\frac{1}{\sqrt{\mu}} D S D .
$$

Note that matrices $D$ and $V$ are symmetric and positive definite. Furthermore, we have

$$
V^{2}=\left(\frac{D^{-1} X D^{-1}}{\sqrt{\mu}}\right)\left(\frac{D S D}{\sqrt{\mu}}\right)=\left(\frac{D^{-1} X S D}{\mu}\right) .
$$

Let us further define

$$
\overline{A_{i}}=\frac{1}{\sqrt{\mu}} D A_{i} D, i=1, \ldots, m, D_{X}=\frac{1}{\sqrt{\mu}} D^{-1} \Delta X D^{-1}, D_{S}=\frac{1}{\sqrt{\mu}} D \Delta S D .
$$

By using (2.11) and (2.13), we find that (2.9) is equivalent to the following system

$$
\left\{\begin{array}{l}
\overline{\mathscr{A}} D_{X}=0, \\
\overline{\mathscr{A}}^{*} \Delta y+D_{S}=0, \\
D_{X}+D_{S}=V^{-1}-V,
\end{array}\right.
$$

where $\overline{\mathscr{A}}$ is the operator defined in (2.6), replacing the matrices $A_{i}$ by $\overline{A_{i}}$ for $i=1, \ldots, m$, and $\overline{\mathscr{A}}^{*}$ is its adjoint. The solution of (2.14) defines the (scaled) $N T$-search direction $\left(D_{X}, \Delta y, D_{S}\right)$. Now, following [12], we replace the right-hand side in the last equation of (2.14) by $-\psi^{\prime}(V)$, where $\psi(V)$ and $\psi^{\prime}(V)$ are defined in Definition 2.2 and Remark 2.3 for our given kernel function $\psi(t)$ in (3.1). Thus we consider the following system

$$
\left\{\begin{array}{l}
\overline{\mathscr{A}} D_{X}=0, \\
\overline{\mathscr{A}}^{*} \Delta y+D_{S}=0, \\
D_{X}+D_{S}=-\psi^{\prime}(V) .
\end{array}\right.
$$

It is easy to verify that this system has a unique solution $\left(D_{X}, \Delta y, D_{S}\right)$. From (2.13), we can compute $\Delta X$ and $\Delta S$. Due to the first two equations of system (2.15), $D_{X}$ and $D_{S}$ are orthogonal, i.e., $\operatorname{tr}\left(D_{X} D_{S}\right)=$ 
$\operatorname{tr}\left(D_{S} D_{X}\right)=0$. Then, we can easily verify that the matrix function $\psi(V)$ determines in a natural way an interior point algorithm

$$
D_{X}=D_{S}=0_{n \times n} \Leftrightarrow \psi^{\prime}(V)=0_{n \times n} \Leftrightarrow V=I \Leftrightarrow \Psi(V)=0 \Leftrightarrow X S=\mu I,
$$

i.e., if and only if $X=X(\mu)$ and $S=S(\mu)$, as it should. Otherwise $\Psi(V)>0$. Thus we conclude that $\Delta X, \Delta y$ and $\Delta S$ all vanish if and only if $V=I$, that is, if and only if $(X, y, S)=(X(\mu), y(\mu), S(\mu))$. Otherwise, we will use $(\Delta X, \Delta y, \Delta S)$ as the new search direction. The new iterate $\left(X_{+}, y_{+}, S_{+}\right)$is given by

$$
X_{+}=X+\alpha \Delta X, y_{+}=y+\alpha \Delta y, S_{+}=S+\alpha \Delta S,
$$

where $\alpha \in] 0,1]$ denotes the default step size. For the analysis of the interior point algorithm, we define the norm-based proximity measure $\sigma$ as follows

$$
\sigma=\frac{1}{2}\left\|D_{X}+D_{S}\right\|=\frac{1}{2}\left\|\psi^{\prime}(V)\right\|=\frac{1}{2} \sqrt{\operatorname{tr}\left(\psi^{\prime}(V)^{2}\right)}=\frac{1}{2} \sqrt{\left\|D_{X}\right\|^{2}+\left\|D_{S}\right\|^{2}} .
$$

\section{NEW PARAMETRIC KERNEL FUNCTION AND ITS PROPERTIES}

In this section, we define our new parametric kernel function and give its properties which are essential to our complexity analysis.

Definition 3.1. $\psi:] 0,+\infty[\rightarrow[0,+\infty[$ is said to be a kernel function if $\psi$ is twice differentiable and the following conditions are satisfied

(1) $\psi^{\prime}(1)=\psi(1)=0$.

(2) $\psi^{\prime \prime}(t)>0, \forall t>0$.

(3) $\lim _{t \rightarrow 0^{+}} \psi(t)=\lim _{t \rightarrow \infty} \psi(t)=\infty$.

For the IPM, we use the following new parametric kernel function

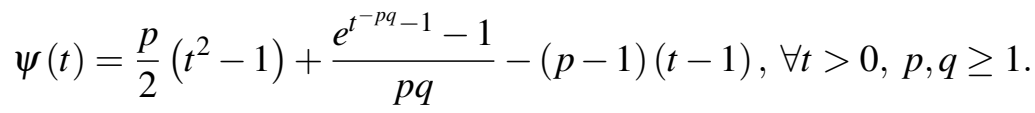

For convenience of reference, we give the first three derivatives of $\psi(t)$ with respect to $t$ as follows

$$
\begin{gathered}
\psi^{\prime}(t)=p t-\frac{e^{t^{-p q}}-1}{t^{p q+1}}-(p-1), \\
\psi^{\prime \prime}(t)=p+\left(p q+(p q+1) t^{p q}\right) \frac{e^{t^{-p q}-1}}{t^{2(p q+1)}}>p \geq 1,
\end{gathered}
$$

and

$$
\psi^{\prime \prime \prime}(t)=-\left[\frac{\left(\frac{p q}{t^{q q}}\right)^{2}+(p q+1)\left[p q+2+\left(\frac{3 p q+1}{t^{p q}}\right)\right]}{t^{p q+3} e^{t^{p q}+1}}\right] .
$$

Furthermore, our new kernel function (3.1) satisfies

$$
\lim _{t \rightarrow 0^{+}} \psi(t)=\lim _{t \rightarrow \infty} \psi(t)=\infty .
$$


Note that $\psi(1)=\psi^{\prime}(1)=0$. Then $\psi(t)$ is determined

$$
\psi(t)=\int_{1}^{t} \int_{1}^{\xi} \psi^{\prime \prime}(\zeta) d \zeta d \xi
$$

The following proposition gives a lower bound of $\sigma$ in terms of $\Psi(V)$.

Proposition 3.2. For any $V \succ 0, \sigma \geq \sqrt{2 p \Psi(V)}, \forall p \geq 1$.

Proof. Since $\psi^{\prime \prime}(t)>p$, we have

$$
\psi(t)=\int_{1}^{t} \int_{1}^{\xi} \psi^{\prime \prime}(\zeta) d \zeta d \xi \leq \frac{1}{p} \int_{1}^{t} \int_{1}^{\xi} \psi^{\prime \prime}(\xi) \psi^{\prime \prime}(\zeta) d \zeta d \xi=\frac{1}{2 p} \psi^{\prime}(t)^{2}, t>0 .
$$

Then,

$$
\sigma^{2}=\operatorname{tr}\left(\psi^{\prime}(V)^{2}\right)=\sum_{i=1}^{n} \psi^{\prime}\left(\lambda_{i}(V)\right)^{2} \geq 2 p \sum_{i=1}^{n} \psi\left(\lambda_{i}(V)\right)=2 p \Psi(V)
$$

Lemma 3.3. [28] Let $\psi(t)$ be a twice differentiable function. Then the following properties are equivalent

(1) $\psi(t)$ is exponentially convex, $\forall t>0$, i.e., $\psi\left(\sqrt{t_{1} t_{2}}\right) \leq \frac{1}{2}\left(\psi\left(t_{1}\right)+\psi\left(t_{2}\right)\right), \forall t_{1}, t_{2}>0$.

(2) $t \psi^{\prime \prime}(t)+\psi^{\prime}(t) \geq 0, \forall t>0$.

Lemma 3.4. Let $\psi(t)$ a kernel function define in (3.1). Then

(1) $\psi(t)$ is convex exponentially.

(2) $\psi^{\prime \prime}(t)$ is decrease monotonically, $\forall t>0$.

(3) $t \psi^{\prime \prime}(t)-\psi^{\prime}(t)>0$.

(4) $\psi^{\prime \prime}(t) \psi^{\prime}(\beta t)-\beta \psi^{\prime}(t) \psi^{\prime \prime}(\beta t)>0, \forall t>1, \beta>1$.

Proof. (1) By Lemma 3.3, it suffices to show that function $\psi(t)$ satisfies $t \psi^{\prime \prime}(t)+\psi^{\prime}(t) \geq 0$, for all $t>0$. Using (3.2) and (3.3), we have

$$
\begin{aligned}
t \psi^{\prime \prime}(t)+\psi^{\prime}(t)= & t\left(p+\left(p q+(p q+1) t^{p q}\right) \frac{e^{t^{-p q}-1}}{t^{2(p q+1)}}\right)+p t-\frac{e^{t^{-p q}-1}}{t^{(p q+1)}}-(p-1) \\
& =(2 t-1) p+1+p q\left(t^{-p q}+1\right) t^{-p q-1} e^{t^{-p q}-1}
\end{aligned}
$$

(a) If $0<t \leq 1$, then

$$
t \psi^{\prime \prime}(t)+\psi^{\prime}(t) \geq 1-p+2 p q=1+p(2 q-1) \geq p+1 .
$$

(b) If $t>1$, then

$$
t \psi^{\prime \prime}(t)+\psi^{\prime}(t)>p+1 .
$$

Hence, $\psi(t)$ is exponentially convex, $\forall t>0$.

(2) From (3.4), we have $\psi^{\prime \prime \prime}(t)<0, \forall t>0$. Then $\psi^{\prime \prime}(t)$ is monotonically decreasing.

(3) From (3.2) and (3.3), we have, for $p, q \geq 1$,

$$
t \psi^{\prime \prime}(t)-\psi^{\prime}(t)=(p-1)+\left(p q t^{-p q}+(p q+2)\right) t^{-p q-1} e^{t^{-p q}-1}>0, \forall t>0 .
$$


(4) If $\psi(t)$ satisfies (2) and (3), we obtain from [28, Lemma 2.4] the result.

This completes the proof.

Lemma 3.5. For $\psi(t)$, we have

(1) $\frac{p}{2}(t-1)^{2} \leq \psi(t) \leq \frac{1}{2 p}\left(\psi^{\prime}(t)\right)^{2}, t>0$.

(2) $\psi(t) \leq \frac{p(2 q+1)+1}{2}(t-1)^{2}, t \geq 1$.

Proof. (1) Using (3.5) and (3.3), we have

$$
\psi(t)=\int_{1}^{t} \int_{1}^{\xi} \psi^{\prime \prime}(\zeta) d \zeta d \xi \geq p \int_{1}^{t} \int_{1}^{\xi} d \zeta d \xi=\frac{p}{2}(t-1)^{2}
$$

which proves the left-hand of inequality. The second hand is obtained as follows

$$
\begin{aligned}
\psi(t) & =\int_{1}^{t} \int_{1}^{\xi} \psi^{\prime \prime}(\zeta) d \zeta d \xi \leq \frac{1}{p} \int_{1}^{t} \int_{1}^{\xi} \psi^{\prime \prime}(\xi) \psi^{\prime \prime}(\zeta) d \zeta d \xi \\
& =\frac{1}{p} \int_{1}^{t} \psi^{\prime \prime}(\xi) \psi^{\prime}(\xi) d \xi=\frac{1}{p} \int_{1}^{t} \psi^{\prime}(\xi) d \psi^{\prime}(\xi) \\
& =\frac{1}{2 p} \psi^{\prime}(t)^{2} .
\end{aligned}
$$

(2) Since $\psi(1)=\psi^{\prime}(1)=0, \psi^{\prime \prime \prime}(t)<0, \forall t>0, \psi^{\prime \prime}(1)=p(2 q+1)+1$, and by using Taylor's theorem, we have

$$
\begin{aligned}
\psi(t) & =\psi(1)+\psi^{\prime}(1)(t-1)+\frac{(t-1)^{2} \psi^{\prime \prime}(1)}{2 !}+\frac{(t-1)^{3} \psi^{\prime \prime \prime}(\xi)}{3 !}, \text { for } 1 \leq \xi \leq t \\
& \leq \frac{1}{2} \psi^{\prime \prime}(1)(t-1)^{2}+\frac{\psi^{\prime \prime \prime}(\xi)}{3 !}(t-1)^{3} \\
& \leq \frac{1}{2} \psi^{\prime \prime}(1)(t-1)^{2},\left(\psi^{\prime \prime \prime}(\xi)<0, \forall \xi \geq 1\right) \\
& \leq \frac{p(2 q+1)+1}{2}(t-1)^{2} .
\end{aligned}
$$

This completes the proof.

Lemma 3.6. Let $\varsigma:[0,+\infty[\rightarrow[1,+\infty[$ be the inverse function of $\psi(t)$, for $t \geq 1$ and let $\rho:[0,+\infty[\rightarrow$ ] $0,1]$ be the inverse function of $-\psi^{\prime}(t)$ for all $\left.\left.t \in\right] 0,1\right]$. Then

(1) $\sqrt{1+\frac{2 S_{\psi}}{p}} \leq \varsigma\left(S_{\psi}\right) \leq 1+\sqrt{\frac{2 S_{\psi}}{p}}$, where $S_{\psi}=\psi(t) \geq 0, p \geq 1$.

(2) $\rho(z) \geq(\ln (z+1)+1)^{-\frac{1}{p q}}, z=-\psi^{\prime}(t) \geq 0$.

Proof. (1) Let $\psi(t)=S_{\psi}, t \geq 1$, i.e., $\varsigma\left(S_{\psi}\right)=t, t \geq 1$. By (3.1), we have

$$
S_{\psi}=\frac{p}{2}\left(t^{2}-1\right)+\frac{e^{t^{-p q}}-1}{p q}-1
$$

which implies that

$$
\frac{p t^{2}}{2}=S_{\psi}+\frac{p}{2}+(p-1)(t-1)+\frac{1-e^{t^{-p q}}-1}{p q} \geq S_{\psi}+\frac{p}{2}
$$

Hence,

$$
t \geq \sqrt{1+\frac{2 S_{\psi}}{p}}
$$


From Lemma 3.5, we have $S_{\psi}=\psi(t) \geq \frac{p}{2}(t-1)^{2}$. So,

$$
t \leq 1+\sqrt{\frac{2 S_{\psi}}{p}}, S_{\psi} \geq 0 .
$$

(2) Let $z=-\psi^{\prime}(t)$, for $\left.\left.t \in\right] 0,1\right]$. Then $\left.\left.\rho(z)=t, t \in\right] 0,1\right]$. Using (3.2), we have

$$
z=-p t+p-1+\frac{e^{t^{-p q}-1}}{t^{p q+1}}
$$

which implies that

$$
\left.\left.e^{t^{-p q}-1} \leq \frac{e^{t^{-p q}-1}}{t^{p q+1}}=z+p t-p+1 \leq z+1, \text { for all } t \in\right] 0,1\right] .
$$

It follows that

$$
t=\rho(z) \geq(\ln (z+1)+1)^{-\frac{1}{p q}}, z \geq 0 .
$$

This completes the proof.

The following lemma is valid for all kernel functions that satisfy (2) and (3) of Lemma 3.4.

Lemma 3.7. [6] Let $\varsigma:[0,+\infty[\rightarrow[1,+\infty[$ be the inverse function of $\psi(t)$, for $t \geq 1$. Then, for any $V \succ 0$ and $\beta>1$, we have

$$
\Psi(\beta V) \leq n \psi\left(\beta \varsigma\left(\frac{\Psi(V)}{n}\right)\right)
$$

We will show in the following corollary that an upper bound for $\Psi\left(\frac{1}{\sqrt{1-\theta}} V\right)$ is expressed with $\Psi(V)$.

Corollary 3.8. Let $\theta$ be such that $0<\theta<1$. Then, for any $V \succ 0$, if $\Psi(V) \leq \tau$, we have

$$
\Psi(\beta V) \leq\left(\frac{p(2 q+1)+1}{2(1-\theta)}\right)\left(\theta \sqrt{n}+\sqrt{\frac{2 \tau}{p}}\right)^{2} .
$$

Proof. Using (2) of Lemma 3.5 for $t \geq 1$, we have

$$
\psi(t) \leq \frac{p(2 q+1)+1}{2}(t-1)^{2}
$$

which implies that

$$
\Psi(\beta V) \leq \frac{n}{2}(p(2 q+1)+1)\left(\beta \varsigma\left(\frac{\Psi(V)}{n}\right)-1\right)^{2} .
$$

From (1) of Lemma 3.6, we have

$$
\begin{aligned}
\Psi(\beta V) & \leq \frac{n}{2}(p(2 q+1)+1)\left(\beta\left(1+\sqrt{\frac{2 \Psi(V)}{n p}}\right)-1\right)^{2} \\
& \leq \frac{1}{2}(p(2 q+1)+1)\left((\beta-1) \sqrt{n}+\beta \sqrt{\frac{2 \Psi(V)}{p}}\right)^{2} .
\end{aligned}
$$

Since $\beta=\frac{1}{\sqrt{1-\theta}}$, we can find

$$
\begin{aligned}
\Psi(\beta V) & \leq\left(\frac{p(2 q+1)+1}{2(1-\theta)}\right)\left((1-\sqrt{1-\theta}) \sqrt{n}+\sqrt{\frac{2 \Psi(V)}{p}}\right)^{2} \\
& \leq\left(\frac{p(2 q+1)+1}{2(1-\theta)}\right)\left(\theta \sqrt{n}+\sqrt{\frac{2 \Psi(V)}{p}}\right)^{2}
\end{aligned}
$$


where the last inequality holds from $1-\sqrt{1-\theta}=\frac{\theta}{1+\sqrt{1-\theta}} \leq \theta$. From the assumption $\Psi(V) \leq \tau$ just before the update of $\mu$, we have

$$
\Psi(\beta V) \leq\left(\frac{p(2 q+1)+1}{2(1-\theta)}\right)\left(\theta \sqrt{n}+\sqrt{\frac{2 \tau}{p}}\right)^{2} .
$$

This completes the proof.

Letting

$$
\Psi_{0}=\left(\frac{p(2 q+1)+1}{2(1-\theta)}\right)\left(\theta \sqrt{n}+\sqrt{\frac{2 \tau}{p}}\right)^{2},
$$

we find that $\Psi_{0}$ is an upper bound for $\Psi(V)$ during the process of the algorithm.

\section{A GENERIC PRIMAL-DUAL INTERIOR POINT ALGORITHM FOR THE SDP}

In general each kernel function gives rise to a primal-dual interior point algorithm. For the description of our algorithm, it is clear that the closeness of $(X, y, S)$ to $(X(\mu), y(\mu), S(\mu))$ is measured by the value of $\Psi(V)$ with $\tau$ as a threshold value. If $\Psi(V) \leq \tau$, then we start a new outer iteration by decreasing $\mu$ to $\mu:=(1-\theta) \mu$, for fixed $\theta \in] 0,1[$. Otherwise, we enter an inner iteration by computing the search direction $(\Delta X, \Delta y, \Delta S)$ at the current iterate with respect to the current value of $\mu$ and apply (2.17) to get new iterate. This process is repeated until $\mu$ is small enough, say until $n \mu<\varepsilon$ for a certain accuracy parameter $\varepsilon$. At this stage, we have found an $\varepsilon$-optimal solution of (P) and (D). The generic form of the algorithm based on our kernel function is given in Algorithm 1.

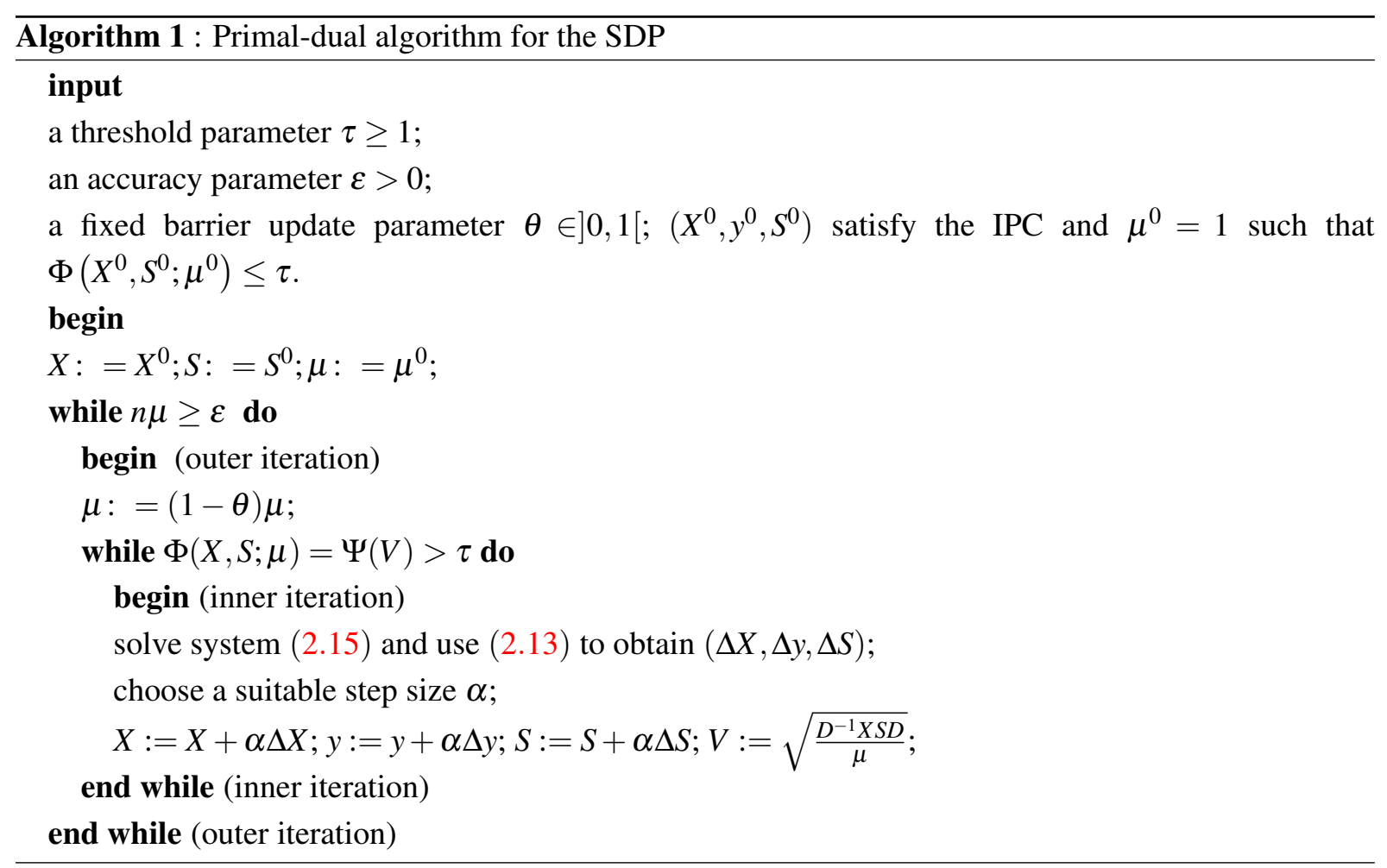

\section{Remark 4.1.}


- The choice of the barrier update parameter $\theta$ plays an important role in both theory and practice of IPMs. Usually, if $\theta$ is a constant independent of the dimension of problem $n$, e.g., $\theta=\frac{1}{2}$, then we call the algorithm a large-update method. If $\theta$ depends on $n$, e.g., $\theta=\frac{1}{\sqrt{n}}$, then the algorithm is called a small-update method.

- The choice of the step size $\alpha$ is another crucial issue in the analysis of the algorithm. It has to be taken such that the closeness of the iterates to the current $\mu$-center improves by a sufficient amount. Each outer iteration consists of an update of parameter $\mu$ and a sequence of (one or more) inner iterations. The total number of inner iterations is the worst-case iteration bound for our algorithm.

\section{ANALYSIS OF THE ALGORITHM}

In this section, we compute a step size such that the proximity function is decreasing and is bound for the decrease during inner iterations and give the complexity results of the algorithm.

5.1. Computing of a default step. In this section, we compute a default step size $\alpha$. After a Newton step, we have

$$
X_{+}=X+\alpha \Delta X, S_{+}=S+\alpha \Delta S .
$$

Using (2.11) and (2.13) for fixed $\mu$, we get

$$
\begin{gathered}
X_{+}=X+\alpha \Delta X=X+\alpha \sqrt{\mu} D D_{X} D=\sqrt{\mu} D\left(V+\alpha D_{X}\right) D \\
S_{+}=S+\alpha \Delta S=S+\alpha \sqrt{\mu} D^{-1} D_{S} D^{-1}=\sqrt{\mu} D^{-1}\left(V+\alpha D_{S}\right) D^{-1} .
\end{gathered}
$$

So, by (2.11), we have $V_{+}=V+\alpha D_{X}=V+\alpha D_{S}$. This implies that the eigenvalues of $V_{+}$are the same as those of the matrix $\left(\left(V+\alpha D_{X}\right)^{\frac{1}{2}}\left(V+\alpha D_{S}\right)\left(V+\alpha D_{X}\right)^{\frac{1}{2}}\right)^{\frac{1}{2}}$. Define, for $\alpha>0, f(\alpha)=\Psi\left(V_{+}\right)-\Psi(V)$. Then, $f(\alpha)$ is the difference of proximities between a new iterate and a current iterate for fixed $\mu$.

Throughout the paper, we assume that the step size $\alpha$ satisfied

$$
V+\alpha D_{X} \succeq 0 \text { and } V+\alpha D_{S} \succeq 0 .
$$

To facilitate the notations, we put $W_{\alpha, X}=V+\alpha D_{X}$ and $W_{\alpha, S}=V+\alpha D_{S}$. From Definition 2.4, we have

$$
\Psi\left(V_{+}\right)=\Psi\left(\left(\left(W_{\alpha, X}\right)^{\frac{1}{2}}\left(W_{\alpha, S}\right)\left(W_{\alpha, X}\right)^{\frac{1}{2}}\right)^{\frac{1}{2}}\right) .
$$

It follows from Proposition 2.5 that

$$
\Psi\left(V_{+}\right) \leq \frac{1}{2}\left(\Psi\left(W_{\alpha, X}\right)+\Psi\left(W_{\alpha, S}\right)\right)
$$

So,

$$
f(\alpha) \leq f_{1}(\alpha)=\frac{1}{2}\left(\Psi\left(W_{\alpha, X}\right)+\Psi\left(W_{\alpha, S}\right)\right)-\Psi(V)
$$

It is obvious that

$$
f(0)=f_{1}(0)=0 .
$$

Taking the first two derivatives of $f_{1}(\alpha)$ with respect to $\alpha$, and using (2.3)-(2.5), we obtain

$$
f_{1}^{\prime}(\alpha)=\frac{1}{2} \operatorname{tr}\left(\psi^{\prime}\left(W_{\alpha, X}\right) D_{X}+\psi^{\prime}\left(W_{\alpha, S}\right) D_{S}\right)
$$


and

$$
f_{1}^{\prime \prime}(\alpha)=\frac{1}{2} \frac{d^{2}}{d \alpha^{2}}\left(\operatorname{tr}\left(\psi\left(W_{\alpha, X}\right)+\psi\left(W_{\alpha, S}\right)\right)\right)
$$

Using $D_{X}+D_{S}=-\psi^{\prime}(V)$ and $\sigma=\sqrt{\left(\operatorname{tr}\left(\left(\psi^{\prime}(V)\right)^{2}\right)\right)}$, we have

$$
f_{1}^{\prime}(0)=-\frac{1}{2} \operatorname{tr}\left(\left(\psi^{\prime}(V)\right)^{2}\right)=-\frac{\sigma^{2}}{2} \text {. }
$$

From three technical Lemmas in [12], we have the following Lemmas 5.1-5.3, since $\psi(t)$ is a kernel function and $\psi^{\prime \prime}(t)$ is monotonically decreasing (see $[6,11,21]$ ).

Lemma 5.1. Let $f_{1}(\alpha)$ be defined as in (5.1) and $\sigma$ be defined as in (2.18). Then

$$
f_{1}^{\prime \prime}(\alpha) \leq \frac{\sigma^{2}}{2} \psi^{\prime \prime}\left(\lambda_{\min }(V)-\alpha \sigma\right)
$$

Lemma 5.2. $f_{1}^{\prime}(\alpha) \leq 0$ holds if $\alpha$ satisfies the inequality

$$
\psi^{\prime}\left(\lambda_{\min }(V)\right)-\psi^{\prime}\left(\lambda_{\min }(V)-\alpha \sigma\right) \leq \sigma .
$$

Lemma 5.3. Let $\rho:[0, \infty[\rightarrow] 0,1]$ be the inverse function of the restriction of $-\psi^{\prime}(t)$. Then, the largest step size $\alpha^{*}$ solution of inequality (5.2) is given as follows

$$
\alpha^{*}=\frac{\rho(\sigma)-\rho(2 \sigma)}{\sigma} .
$$

For the purpose of finding an upper bound of $f(\alpha)$, we need a default step size $\bar{\alpha}$, which is the lower bound of the $\alpha^{*}$ and consists of $\sigma$.

Lemma 5.4. Let $\rho:[0, \infty[\rightarrow] 0,1]$ be the inverse function of the restriction of $-\psi^{\prime}(t)$ in the interval $\left.] 0,1\right]$ and let $\alpha^{*}$ be defined as in (5.3). Then

$$
\alpha^{*} \geq \frac{1}{p+(2 p q+1)(1+2 \sigma) t^{-p q-1}} .
$$

Proof. From $-\psi^{\prime}(\rho(\sigma))=\sigma$, and taking the derivative of $\sigma$ at both sides, we get

$$
\rho^{\prime}(\sigma)=\frac{-1}{\psi^{\prime \prime}(\rho(\sigma))}
$$

Since $\psi^{\prime \prime}>0, \rho(\sigma)$ is monotonically decreasing in $\sigma$, we have

$$
\alpha^{*}=\frac{1}{\sigma}(\rho(\sigma)-\rho(2 \sigma))=\frac{1}{\sigma} \int_{2 \sigma}^{\sigma} \rho^{\prime}(\xi) d \xi \geq \frac{1}{\sigma}\left[\frac{\xi}{\psi^{\prime \prime}(\rho(2 \sigma))}\right]_{\sigma}^{2 \sigma}=\frac{1}{\psi^{\prime \prime}(\rho(2 \sigma))}=\tilde{\alpha}
$$

where the inequality follows from $\sigma \leq \xi \leq 2 \sigma$ with $\rho$ and $\psi^{\prime \prime}$ are monotonically decreasing. The inverse function of $\left.\left.-\psi^{\prime}(t), t \in\right] 0,1\right]$ is hard to solve, so we should derive a lower bound for $\rho(S)$. From $\psi^{\prime}(t)=p t-(p-1)-e^{t^{-p q}-1} t^{-p q-1}$, let $\left.\left.S_{b}=-\psi_{b}^{\prime}(t)=e^{t^{-p q}-1} t^{-p q-1}:\right] 0,1\right] \rightarrow[1, \infty[$ and let $\rho_{b}:[1, \infty[\rightarrow] 0,1]$ denote the inverse function of the restriction of $-\psi_{b}^{\prime}(t)$ to the interval $\left.] 0,1\right]$. Then

$$
S_{b}=e^{\rho_{b}\left(S_{b}\right)^{-p q}-1} \rho_{b}\left(S_{b}\right)^{-p q-1},
$$

where

$$
\rho_{b}\left(S_{b}\right)=t, S_{b} \geq 1,0<\rho_{b}\left(S_{k}\right) \leq 1
$$


This means that

$$
e^{\rho_{b}\left(S_{b}\right)^{-p q}-1}=S_{b} \rho_{b}\left(S_{b}\right)^{p q+1} \leq S_{b} .
$$

Hence

$$
\rho_{b}\left(S_{b}\right)=t \geq \frac{1}{\left(1+\ln S_{b}\right)^{\frac{1}{p q}}} .
$$

Letting $-\psi^{\prime}(t)=S$, we have

$$
-S=p t-p+1+\psi_{b}^{\prime}(t), \quad 0<t \leq 1 .
$$

So,

$$
-\psi_{b}^{\prime}(t)=p t-p+1+S \leq 1+S .
$$

Since $-\psi_{b}^{\prime}(t)$ is monotonically decreasing, and $\rho_{b}\left(S_{b}\right)$ is also monotonically decreasing, we say that

$$
\rho(S)=t=\rho_{b}\left(S_{b}\right)=\rho_{b}\left(-\psi_{b}^{\prime}(t)\right) \geq \rho_{b}(1+S) .
$$

Here, let $t=\rho_{b}(1+2 \sigma)$. Then $-\psi_{b}^{\prime}(t)=1+2 \sigma$. Using the definition of $\psi^{\prime \prime}(t)$ with $t=\rho(2 \sigma)$ and $S_{b}=e^{t^{-p q}-1} t^{-p q-1}=1+2 \sigma$, we can obtain

$$
\tilde{\alpha} \geq \frac{1}{p+(2 p q+1)(1+2 \sigma) t^{-p q-1}} .
$$

For using $\bar{\alpha}$ as the default step size in the algorithm, we define the $\bar{\alpha}$ as follows

$$
\bar{\alpha}=\frac{1}{p+(2 p q+1)(1+2 \sigma) t^{-p q-1}} .
$$

5.2. Decreasing of the proximity function during a Newton step. Now, we show that our proximity function $\Psi$ with our default step size $\bar{\alpha}$ is decreasing. It can be easily established by using the following result.

Lemma 5.5. [6] Let $h$ be a twice differentiable convex function with $h(0)=0, h^{\prime}(0)<0$, which attains its (global) minimum at $t^{*}>0$. If $h^{\prime \prime}$ is increasing for $t \in\left[0, t^{*}\right]$, then

$$
h(t) \leq \frac{t h^{\prime}(0)}{2}, 0 \leq t \leq t^{*}
$$

The upper bound for the decreasing value of the proximity in the inner iteration is given in the following theorem.

Theorem 5.6. Let $\bar{\alpha}$ be a step size defined as in (5.6) and $\sigma \geq 1$. Then

$$
f(\bar{\alpha}) \leq-\frac{\Psi^{\frac{1}{2}}}{2+6 \sqrt{2}(2 p q+1)\left(1+\ln 3 \sqrt{2 p} \sqrt{\Psi_{0}}\right)^{1+\frac{1}{p q}}} .
$$

Proof. Since $f_{1}^{\prime}(0)=f_{2}^{\prime}(0)=-\frac{\sigma^{2}}{2}$ and $\bar{\alpha} \in\left[0, \alpha^{*}\right]$, we have

$$
\begin{aligned}
f(\bar{\alpha}) & \leq-\frac{\sigma^{2}}{4} \bar{\alpha}=-\frac{1}{4} \cdot \frac{\sigma^{2}}{p+(2 p q+1)(1+2 \sigma) t^{-p q-1}} \\
& \leq-\frac{1}{4} \cdot \frac{\sigma^{2}}{p+3 \sigma(2 p q+1)(1+\ln 3 \sigma)^{1+\frac{1}{p q}}}(\text { put } t=\varsigma(1+2 \sigma) \text { in }(5.5)) .
\end{aligned}
$$


This expresses the decrease in one inner iteration in terms of $\sigma$. Since the decrease depends monotonically on $\sigma$, we can express the decrease in terms of $\Psi=\Psi(V)$ from proposition 3.2 as follows

$$
\begin{aligned}
f(\bar{\alpha}) & \leq-\frac{1}{4} \cdot \frac{2 p \Psi}{p+3 \sqrt{2 p \Psi(2 p q+1)(1+\ln 3 \sqrt{2 p \Psi})^{1+\frac{1}{p q}}}} \\
& \leq-\frac{1}{2} \cdot \frac{\Psi^{\frac{1}{2}}}{1+\frac{3 \sqrt{2}}{\sqrt{p}}(2 p q+1)\left(1+\ln 3 \sqrt{2 p} \sqrt{\Psi_{0}}\right)^{1+\frac{1}{p q}}} \\
& \leq-\frac{1}{2} \cdot \frac{\Psi^{\frac{1}{2}}}{1+3 \sqrt{2}(2 p q+1)\left(1+\ln 3 \sqrt{2 p} \sqrt{\Psi_{0}}\right)^{1+\frac{1}{p q}}},
\end{aligned}
$$

where the second inequality follows from $\Psi_{0} \geq \Psi \geq \tau \geq 1$. This completes the proof.

5.3. Iteration complexity. In this subsection, we need to count how many inner iterations are required to return to the situation where $\Psi(V) \leq \tau$ after $\mu$-update. At this stage, we invoke the following lemma.

Lemma 5.7. $[6,12]$ Let $t_{0}, t_{1}, \ldots, t_{k}$ be a sequence of positive numbers such that

$$
t_{k+1} \leq t_{k}-\beta t_{k}^{1-\gamma}, k=0,1, \ldots, K-1,
$$

where $\beta>0$ and $0<\gamma \leq 1$. Then

$$
K \leq\left[\frac{t_{0}^{\gamma}}{\beta \gamma}\right]
$$

Lemma 5.8. Let $K$ be the total number of inner iterations in the outer iteration. Then,

$$
K \leq\left[2 K_{\max } \Psi_{0}^{\frac{1}{2}}\right]
$$

where $K_{\max }=\left(2+6 \sqrt{2}(2 p q+1)\left(1+\ln 3 \sqrt{2 p} \sqrt{\Psi_{0}}\right)^{1+\frac{1}{p q}}\right)$ and $\Psi_{0}$ is the value of $\Psi(V)$ after the $\mu$ update in outer iteration.

Proof. Using Lemma 5.7 for $t_{k}=\Psi_{k}, \beta=\frac{1}{\left(2+6 \sqrt{2}(2 p q+1)\left(1+\ln 3 \sqrt{2 p} \sqrt{\Psi_{0}}\right)^{1+\frac{1}{p q}}\right)}$ and $\gamma=\frac{1}{2}$, we have

$$
\Psi_{k+1} \leq \Psi_{k}-\frac{\Psi_{k}^{\frac{1}{2}}}{2+6 \sqrt{2}(2 p q+1)\left(1+\ln 3 \sqrt{2 p} \sqrt{\Psi_{0}}\right)^{1+\frac{1}{p q}}} .
$$

This completes the proof.

Now, we estimate the total number of iterations of our algorithm.

Theorem 5.9. Let $\Psi_{0}$ be defined in (3.6) and $\tau \geq 1$. Then, the total number of iterations required by the algorithm to obtain an approximation solution with $n \mu \leq \varepsilon$ is bounded by

$$
\left[2 K_{\max } \Psi_{0}^{\frac{1}{2}}\right]\left[\frac{1}{\theta} \ln \frac{n}{\varepsilon}\right] .
$$

Proof. The number of outer iterations is the number of iterations $k$ necessary to obtain $n \mu \leq \varepsilon$. Previous and new $\mu$ are related as follows $\mu:=(1-\theta) \mu$. Thus, $n \mu \leq \varepsilon$ can be written as $(1-\theta)^{k} \mu_{0} \leq \frac{\varepsilon}{n}$. We assume that $\mu_{0}=1$. By taking the algorithm of both sides of the inequality, we obtain $k \ln (1-\theta) \leq \ln \frac{\varepsilon}{n}$. Using the Taylor theorem for $\ln (1-\theta)$, we obtain $k \leq \frac{1}{\theta} \ln \frac{n}{\varepsilon}$. Therefore, the number of outer iterations is bounded above by $\frac{1}{\theta} \ln \frac{n}{\varepsilon}$. Multiplication of this result by the number in the above Lemma yields this theorem 
For large-update method with $\tau=\mathscr{O}(n)$ and $\theta=\Theta(1)$, we get $\Psi_{0}=\mathscr{O}(n)$ and $\mathscr{O}\left(\sqrt{n}(\ln n)^{\frac{p q+1}{p q}} \ln \frac{n}{\varepsilon}\right)$ iterations complexity.

For small-update with $\tau=\mathscr{O}(1)$ and $\theta=\Theta\left(\frac{1}{\sqrt{n}}\right)$, we get $\Psi_{0}=\mathscr{O}(1)$ and $\mathscr{O}\left(\sqrt{n} \ln \frac{n}{\varepsilon}\right)$ iterations bound.

\section{COMPARISON OF ALgORITHMS}

In this section, we offer a comparison between the kernel function proposed by Choi and Lee [12] with exponential barrier term and our kernel function. We summarize the results of their correspondent IPMs algorithms in tables 1, 2 and 3.

1) The first kernel function is defined as follows

$$
\psi(t)=\frac{1}{2}\left(t^{2}-1\right)+\frac{e^{t^{-q}-1}-1}{q}, \forall t>0, q \geq 1 .
$$

2) Our new kernel function defined in (3.1)

$$
\psi(t)=\frac{p}{2}\left(t^{2}-1\right)+\frac{e^{t^{-p q}-1}-1}{p q}-(p-1)(t-1), \forall t>0, p, q \geq 1 .
$$

TABLE 1. Properties of kernel functions

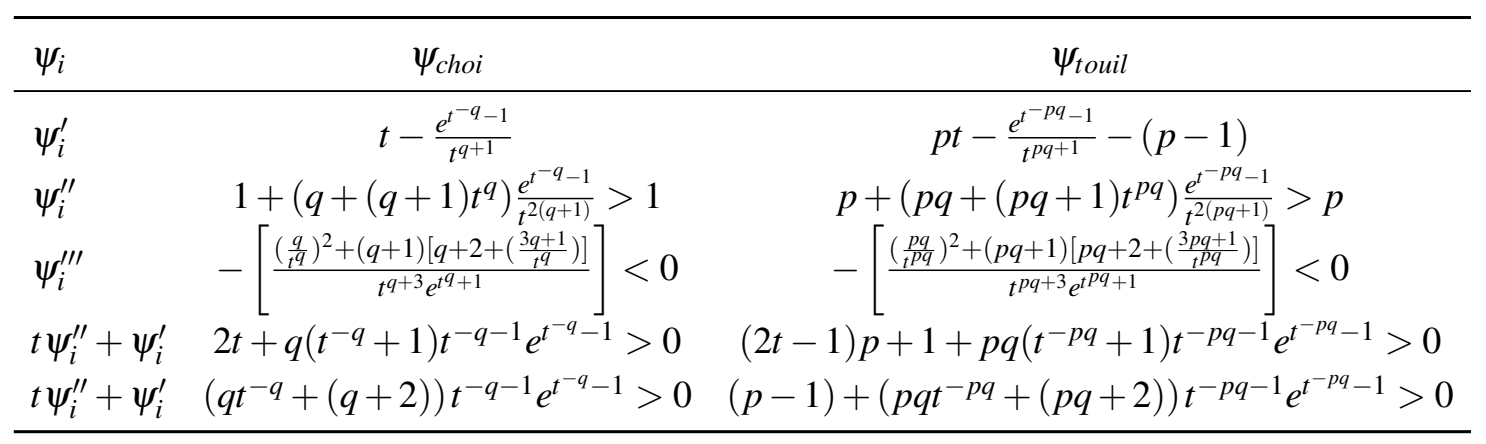

TABLE 2. The estimated bound terms of the algorithm

\begin{tabular}{lcc}
\hline$\psi_{i}$ & $\psi_{\text {choi }}$ & $\psi_{\text {touil }}$ \\
\hline$\varsigma\left(S_{\psi}\right)$ for $S_{\psi} \geq 0$ & $\sqrt{1+2 S_{\psi}} \leq \varsigma\left(S_{\psi}\right) \leq 1+\sqrt{2 S_{\psi}}$ & $\sqrt{1+\frac{2 S_{\psi}}{p}} \leq \varsigma\left(S_{\psi}\right) \leq 1+\sqrt{\frac{2 S_{\psi}}{p}}$ \\
$\rho(z)$ for $z \geq 0$ & $\rho(z) \geq(\ln (z+1)+1)^{-\frac{1}{q}}$ & $\rho(z) \geq(\ln (z+1)+1)^{-\frac{1}{p q}}$ \\
$\Psi_{0}$ & $\left(\frac{(2 q+1)+1}{2(1-\theta)}\right)(\theta \sqrt{n}+\sqrt{2 \tau})^{2}$ & $\left(\frac{p(2 q+1)+1}{2(1-\theta)}\right)\left(\theta \sqrt{n}+\sqrt{\frac{2 \tau}{p}}\right)^{2}$ \\
$\bar{\alpha}$ & $\frac{1}{1+(2 q+1)(1+2 \sigma) t^{-q-1}}$ & $\frac{1}{p+(2 p q+1)(1+2 \sigma) t^{-p q-1}}$ \\
$\beta$ & $\left(2+6 \sqrt{2}(2 q+1)\left(1+\ln 3 \sqrt{2 \Psi_{0}}\right)^{1+\frac{1}{q}}\right)$ & $\frac{1}{\left(2+6 \sqrt{2}(2 p q+1)\left(1+\ln 3 \sqrt{2 p} \sqrt{\Psi_{0}}\right)^{1+\frac{1}{p q}}\right)}$ \\
$\gamma$ & $\frac{1}{2}$ & $\frac{1}{2}$ \\
\hline
\end{tabular}


TABLE 3. Complexity bounds for both large and small-update methods

\begin{tabular}{lcc}
\hline$\psi_{i}$ & $\psi_{\text {choi }}$ & $\psi_{\text {touil }}$ \\
\hline Large-update methods & $\mathscr{O}\left(\sqrt{n}(\ln n)^{\frac{q+1}{q}} \ln \frac{n}{\varepsilon}\right)$ & $\mathscr{O}\left(\sqrt{n}(\ln n)^{\frac{p q+1}{p q}} \ln \frac{n}{\varepsilon}\right)$ \\
Small-update methods & $?$ & $\mathscr{O}\left(\sqrt{n} \ln \frac{n}{\varepsilon}\right)$ \\
\hline
\end{tabular}

\section{CONCLUSIONS AND REMARKS}

In this paper, we proposed a class of primal-dual IPMs for the SDP based on a new parametric kernel function, which is a combination between a growth term and an exponential barrier term. We analyzed the primal-dual interior point algorithm based on this kernel function, and established that the worst-case iteration bounds are $\mathscr{O}\left(\sqrt{n}(\ln n)^{\frac{p q+1}{p q}} \ln \frac{n}{\varepsilon}\right)$ and $\mathscr{O}\left(\sqrt{n} \ln \frac{n}{\varepsilon}\right)$ for large and small-update methods, respectively. Our results improve the complexity results obtained in $[7,12,21]$ for large-update methods. We also remark that the extensions to second-order cone programming (SOCP), and symmetric cone programming (SCP) deserves to be further investigated. Furthermore, the numerical results may help us compare the behavior of the proposed algorithms with existing IPMs.

\section{REFERENCES}

[1] N. Karmarkar, A new polynomial-time algorithm for linear programming, Combinatorica 4 (1984), 373-395.

[2] M. Achache, A weighted path-following method for the linear complementarity problem, Studia Universitatis BabesBolyai Series Informatica 49 (2004), 61-73.

[3] Z. Kebbiche, D. Benterki, A weighted path-following method for linearly constrained convex. Revue Roumaine de Mathématique Pures et Appliquees, 57 (2012), 245-256.

[4] M.S. Babynin, V.G. Zhadan, A primal interior point method for the linear semidefinite programming problem, Comput. Math. Math. Phys. 48 (2008), 1746-1767.

[5] I. Touil, D. Benterki, A. Yassine, A feasible primal-dual interior point method for linear semidefinite programming, J. Comput. Appl. Math. 312 (2017), 216-230.

[6] G.Q. Wang, Y.Q. Bai, C. Roos, Primal-dual interior-point algorithms for semidefinite optimization based on a simple kernel function, J. Math. Model. Algo. 4 (2005), 409-433.

[7] M. El Ghami, I.D. Ivanov, C. Roos, T. Steihaug, A polynomial-time algorithm for LO based on generalized logarithmic barrier functions, Int. J. Appl. Math. 21 (2008), 99-115.

[8] C. Roos, T. Terlaky, J. Vial, Theory and Algorithms for Linear Optimization, in: An interior point approach, John Wiley \& Sons, Chichester, UK, 1997.

[9] J. Peng, C. Roos, T. Terlaky, Self-regular functions and new search directions for linear and semidefinite optimization, Math. Program. 93 (2002), 129-171.

[10] J. Peng, C. Roos, T. Terlaky, Self-regularity: A new Paradigm for Primal-Dual Interior-Point Algorithms, Princeton University Press, Princeton, (2002).

[11] G.M. Cho, An interior-point algorithm for linear optimization based on a new barrier function, Appl. Math. Comput. 218 (2011), 386-395.

[12] B.K. Choi, G.M. Lee, On complexity analysis of the primal-dual interior-point method for semidefinite optimization problem based on a new proximity function, Nonlinear Anal. 71 (2009), 2628-2640.

[13] Y.Q. Bai, C. Roos, A primal-dual interior-point method based on a new kernel function with linear growth rate, Proceedings of Industrial Optimization Symposium and Optimization Day, Australia, 2002.

[14] Z.G. Qian, Y.Q. Bai, G.Q. Wang, Complexity analysis of interior-point algorithm based on a new kernel function for semi-definite optimization, J. Shanghai Univ. 12 (2008), 388-394.

[15] M.W. Zhang, A large-update interior-point algorithm for convex quadratic semi-definite optimization based on a new kernel function, Acta Mathematica Sinica, 28 (2012), 2313-2328. 
[16] X.Z. Cai, G.Q. Wang, M. El Ghami, Y.J. Yue, Complexity analysis of primal-dual interior-point methods for linear optimization based on a new parametric kernel function with a trigonometric barrier term, Abstr. Appl. Anal. Art. 11 (2014), Article ID 710158.

[17] M.R. Peyghami, S.F. Hafshejani, L. Shirvani, Complexity of interior point methods for linear optimization based on a new trigonometric kernel function, J. Comput. Appl. Math. 255 (2014), 74-85.

[18] M.R. Peyghami, S.F. Hafshejani, Complexity analysis of an interior point algorithm for linear optimization based on a new proximity function, Numer. Algo. 67 (2014), 33-48.

[19] M.R. Peyghami, S. Fathi-Hafshejani, S. Chen, A primal-dual interior-point method for semidefinite optimization based on a class of trigonometric barrier functions, Oper. Res. Lett. 44 (2016), 319-323.

[20] X. Li, M. Zhang, Interior-point algorithm for linear optimization based on a new trigonometric kernel function, Oper. Res. Lett. 43 (2015), 471-475.

[21] M. Bouafia, D. Benterki, A. Yassine, Complexity analysis of interior point methods for linear programming based on a parameterized kernel function, RAIRO Oper. Res. 50 (2016), 935-949.

[22] R.A. Horn, C.R. Johnson, Topics in Matrix Analysis, Cambridge University Press, Cambridge, 1991.

[23] H. Lütkepohl, Handbook of Matrices, Humboldt-Universität zu Berlin, , 1996.

[24] H. Wolkowicz, R. Saigal, L. Vandenberghe, Handbook of Semidefinite Programming, Theory, Algorithms, and Applications, Kluwer Academic Publishers, Boston, 2000.

[25] C. Helmberg, F. Rendl, R.J. Vanderbei, H. Wolkowicz, An interior-point method for semidefinite programming, SIAM J. Optim. 6 (1996), 342-361.

[26] M.J. Todd, K. C. Toh, Tütüncü, On the nestrov-todd direction in semidefinite programming, SIAM J. Optim. 8 (1998), 769-796.

[27] Y. Zhang, On extending some primal-dual algorithms from linear programming to semidefinite programming, SIAM J. Optim. 8 (1998), 365-386.

[28] Y.Q. Bai, M. El Ghami, C. Roos, A comparative study of kernel functions for primal-dual interior-point algorithms in linear optimization, SIAM J. Optim. 15 (2004), 101-128. 